\title{
Secured Vehicle Safety System using GSM Technology
}

\author{
A.Swapna, J.T Arun Kumar
}

\begin{abstract}
A smart helmet is a kind of defensive headgear utilized by the rider which makes bike driving more secure than previously. The principle reason for this keen protective cap to give well being to rider.Here I proposed a work which is endeavor to plan a propelled vehicle's security framework which utilizes GSM to avert burglary and to decide the area of vehicles. Now a daysburglary is going on the stopping or in some shaky spots. The wellbeing of the vehicles is incredibly fundamental. The point of the vehicles security framework is used to utilizes the remote communication innovatively for the car situations. The principle focal point of this undertaking is to ensure the stealing of vehicle. This is finished with the assistance of GSM modem and circuit which comprises of ARM 7 TDMI microcontroller, transfer and venture down transformer. The framework will be enacted simply in the wake of wearing the head protector or else the client can't ready to get to the vehicle. To achieve Automated Vehicle Location our system uses to transmit the area data continuously, Active systems are produced. Progressing vehicular after system joins a gear device introduced in the vehicle and a remote Tracking servers. The infowas conveyed to Tracking server utilizing GSM/GPRS modem on GSM mastermind by using SMS or utilized direct TCP/IP association with Tracking servers thruGPRS. Following servers in like way has GSM/GPRS modem that gets vehicle region data by techniques for GSM system and stores info into databases. This info is available to embraced clients of the systems by techniques for sites over the web.
\end{abstract}

\section{INTRODUCTION}

Now a day's vehicle theft cases are higher than later, it had the option to be chief to give a vehicle an extraordinary securities with the crucial solid threatening to break-in gadget. Vehicle principal locking structure guarantees the best assurance to grapple your vehicle from numerous sorts of burglary cases. Right now GPS vehiclesare following guarantees their flourishing as voyaging.

Vehicle Security System depends on GSM module. An appropriate report and usage of this task can be utilized for different applications. It is an exemplary case of remote communications. The remote interchanges industry is one of the quickest developing ventures. In the course of recent years, there has been an unstable increment in the robbery of vehicles. With the assistance of investigation of GSM, robbery implication was absolute originally imagined in Spain and United Kingdom.

The underlying foundations of Vehicle Tracking Systems lies in transportation industries. At the point when extensive armada of vehicles were spread outover the immense breadths of sea, the proprietor enterprises regularly thought that it was hard to monitor what was going on. They

Revised Manuscript Received on April 12, 2019.

A.Swapna, Department of Electronics and Communication Engineering,SR Engineering College, Warangal, Telangana, India

Dr.J.Tarun Kumar, Department of Electronics and Communication Engineering, SR Engineering College, Warangal, Telangana, India required a type of framework to trace out every vehicleand it has some random time and for to what extent it voyaged.

\section{LITERATURE SURVEY}

Prajitha Prasad A, Reshma Mohan, et al.,(2018)The basic target of this paper is to construct a well being system which is coordinated with the savvy protective cap and keen bicycle to lessen the likelihood of bike mishaps and alcoholic drive cases. The bicycle will begin till the rider attires the head protector and if there is no alcoholic substance presents. At that point when the rider lays down, head protector hits the ground, then sensors identify the movement and slopes of cap and reports the event of a mishap. It sends data of the comparing area to relatives of the rider and crisis contact number.

Bhumi Bhatt, PurviKalani, et al.,(2015)The crucial precept of endeavor is to use the remote development reasonably for the vehicle circumstances by means of the GSM Modem used in sending SMS in cases of burglary indication. Here essential degree of this undertaking is to stop vehicle engine normally. The GSM modem offers info to the client on his mentioning. The proprietor can gets the situation of vehicle at any moment. She/he bestows somewhat express to shock the vehicle. The GPS beneficiary on their unit will find the degree and longitude of the vehicle make use the satellite association.

\section{TECHNIQUES USED}

\section{GSM MODULE}

GSM may be a progressed versatile correspondence structure that's used the globe over. GSM uses a range of your time division numerous access (TDMA) and it is most of the foremost half used of the 3 machine-controlled remote correspondence advancements (TDMA, GSM, and CDMA). GSM digitizes and packs info, by it sends down to a channel with 2 distinctive deluges of client info, whenever providing gap. It works at 850, 900, 1800, 1900 and 2100 rate likewise another repeat gatherings.

Modem may be a Tri-band GSM/GPRS engine wears down frequencies EGSM $900 \mathrm{MHz}$, DCS $1800 \mathrm{MHz}$ and PCS $1900 \mathrm{MHz}$. SIM three hundred options GPRS multispace category 10/class eight (optional) and it supports the GPRS secret writing plans CS-1, CS-2, CS-3 and CS-4. AT Commands will be accustomed get data in SIM card. SIM interface supports the accessibility of the GSM Phase1 assurance and besides supports the convenience of the new GSM Phase2 + detail for quick sixty four kbps SIM

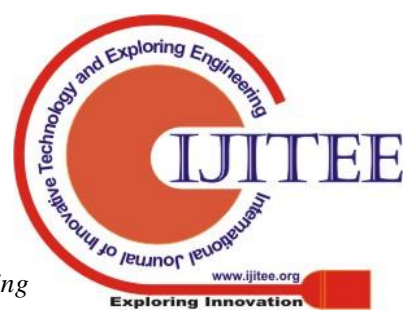


(expected to be used with a SIM application Tool-pack). Both $1.8 \mathrm{~V}$ and 3.0V SIM Cards area unit bolstered. SIM interface is energized from an interior controller within the module having apparent voltage two.8V.TheGSM electronic equipment is equipped a sim-plate within which a sim is to be embedded.

Network status indication LED lamp

StateSIM300function

Off-SIM300 isn't running

$64 \mathrm{msOn} / 0.8 \mathrm{secOff}$ - SIM300 does not discover the system

64msOn/3SecOff - SIM300 discover the system

$64 \mathrm{msOn} / 0.3 \mathrm{secOff}-\mathrm{GPRS}$ communication

\section{GPS MODULE}

The Global Positioning System (GPS) may be a satellitebased routing system created from a system of twenty four satellites set into house by the U.S.A. GPS works in any climatic conditions, on the earth, $24 \times 7$. GPS satellites circle the world twofold per day in a precise circle and transmit flags knowledge to earth. A GPS recipient takes this knowledge and use triangulation to reckon the client's correct space. primarily, the GPS beneficiary dissects the time a banner was transmitted by a satellite with the time it had been gotten. The time qualification tells the GPS recipient however distant the satellite is directly, with detachment estimations from 2 or 3 further satellites, the beneficiary will opt for the customer's position and show it on the unit's electronic guide. A GPS beneficiary should be broken on to the banner of no beneath 3 satellites to register a 2nd position (degree and longitude) and track advancement. Here in any event 4 satellites in sight, the recipient will opt for the customer's 3D position.

\section{CIRCUIT DIAGRAM}

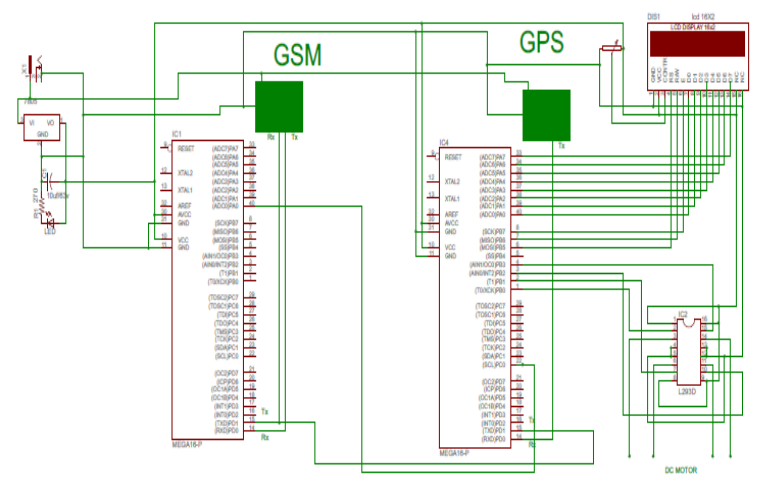

Circuit diagram of GSM and GPS

\section{RF Communication circuit}

Helmet unit and bike unit are are associated by remote connection of RF. Encoder is protective cap inside which is utilized to change over parallel information into sequential information. The encoder is fit for encoding message which contains of $12 \mathrm{~N}$ information bits and $\mathrm{N}$ address bit. Each location/information can remain set to with two consistent states. The oscillator recurrence is $3 \mathrm{kHz}$, with rosc of $1 \mathrm{M}$ ohms. Least transmission of information is 4 words. Decoder is on bicycle side, it is utilized to interpret sequential information. It convert this sequential information into parallel. The decoders are fit for get information that are spread by an encoder and get it. The main piece time frame use as addresses and last $12 \mathrm{~N}$ bits as our ideal information, $\mathrm{N}$ is represents address number.

Block Diagram

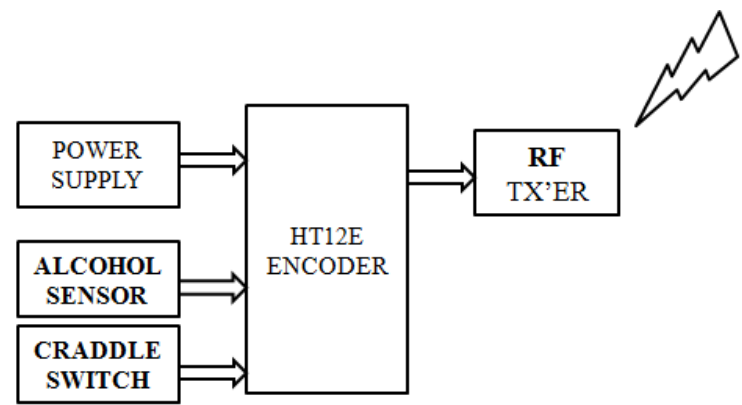

Helmet section block diagram

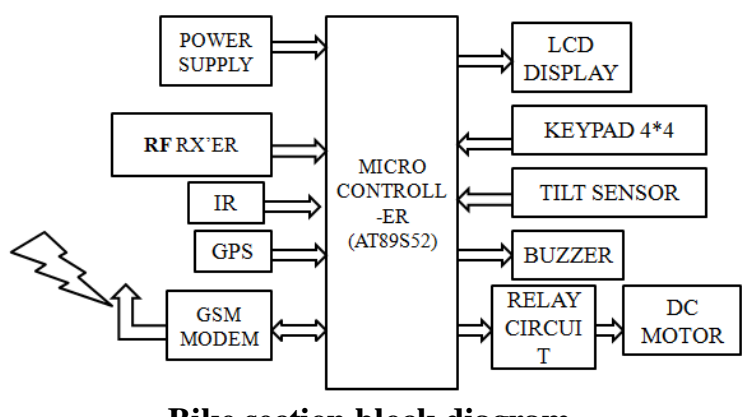

Bike section block diagram

Block diagram consists of 2-units Helmet unitand Bike unit.

Helmet unit consist of controller, alcohol sensor, RF TX'ER, power supply and cradle switch and the bike section consists of GSM, GPS, RF TX'ER, Tilt sensor, Relay circuit, IR sensor, DC motor, buzzer keypad, LCD display.

\section{SYSTEM OVERVIEW}

\section{Power supply}

The Power Supply is a Main necessity for any task work. The required DC power source for the base unit just as the energizing unit is gotten from the mains line..

\section{GPS}

Global Positioning System(GPS) development is changing the manner by which we work and play. You can use the GPS improvement when you are driving, figuring, cruising, climbing, running, biking, working, or investigating. With a GPS expert, you have a dumbfounding extent of info expeditiously open.

\section{GSM}

Global System for Mobile Communication (GSM) is a ton of ETSI rules deciding the structure for a mechanized cell organization. Here Modem is going with RS232 interfaces, grants you partner PC similarly as microcontroller with RS232 Chip(MAX232).

Its works in the frequency $(800 \mathrm{MHz})$ of our portable correspondence range $(800-1200 \mathrm{MHz})$.

Published By: 


\section{RF Tx'er\& RF Rx'er}

This pair of collector.transmitter modules are valuable for short separation ( 5-6 meters) remote information transmission/gathering and control. These modules give a minimal effort mean of including remote component into your electronic items. They take a shot at $433 \mathrm{MHz}$ transporter and utilizations ASK computerized tweak procedure to stream information more than $433 \mathrm{MHz}$ bearer.

\section{Buzzer}

A beeper is a sound flagging gadget, which might be mechanical, electromechanical, or piezoelectric.

\section{Alcohol sensor}

The sensor MQ-2 is Gas/Smoke sensor. This is assigned to LPG, Hydrogen, Smoke, Methane, Propane, Alcohol, Butane and some other mechanical flammable gases.

\section{IR \& Tilt Sensor}

This Reflective IR Sensor is major plastic packaging with 2-portions an IR LED and an IR phototransistor. You can regulate the IR LED and turn it on to ricochet IR off things to pick their reflectivity. White and light tinted stuff will influence the light, so you can recall it. Diminish and dull concealed stuff will ingest the IR light with the target isn't seen. Also, if something isn't disheartening the sensors, it won't trigger either.

\section{DC mortor}

A DC engine depends upon the way wherein that like magnet shafts repulse and in no way like engaging posts pull in one another. A spot of wire with a present encountering it conveys an electromagnetic field concurred with the purpose of assembly of the circle. By exchanging the current on or off in a wind its charming field can be turned on or off or by exchanging the course of the present insider shrewd the heading of the conveyed engaging field can be exchanged $180^{\circ}$.

\section{Motor driver}

It is a driver which is utilized to drive the engine dc by utilizing transistor rationale.

\section{Keypad (4X4)}

Keypad $4 \times 4$ is used for stacking numerics into the microcontroller. It includes 16 gets planned in a kind of a group containing four lines and four fragments. It is related with the improvement system by standard IDC 10 female connector associated some headway structure's port.

This 16-get keypad gives a profitable human interface part for microcontroller adventures. Accommodating bond bolster gives a direct technique to mount the keypad in a variety of usages.LCD display (16X2)

\section{SOFTWARE USED:}

\section{ABOUT KEIL Software}

It is possible to form the deliver documents in anexceedingly matter content editor like tablet, run the Compiler on each $\mathrm{C}$ provide file, specifying an inventory of controls, run the program on each program supply file, specifying each different listing of controls, run each the
LibraryManager or Linker (again specifying a listing of controls) associate degreed later walking the ObjectHEX device to convert the Linker output document to an Intel Hex File. Once that has been completed the Hex File is also downloaded to the aim hardware and debugged. or else KEIL is wont

to produceprovidefiles;automaticallycollect, link associate degreed covertexploitationalternatives set with an swish to use person interface and sooner or later simulate or do debugging on the hardware with get right of entry to $\mathrm{C}$ variables and memory. Unlessyou would like to use the tolls on the statement, the selection is evident. KEIL Greatlysimplifies the technique of constructing associate degreed making an attempt out an embedded package.

\section{RESULTS}

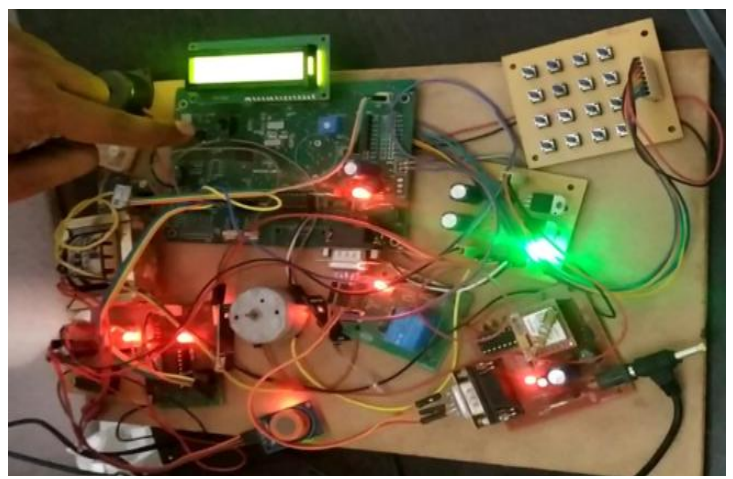

The results shown in the figure was received from anti theft system for vehicle security which is IR Based GSM and GPS in this I was used continues vehicle tracking.

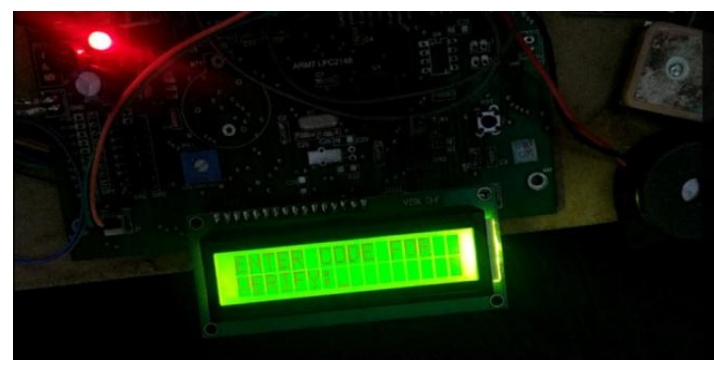

If the rider starts the bike then it is display as the verify your code

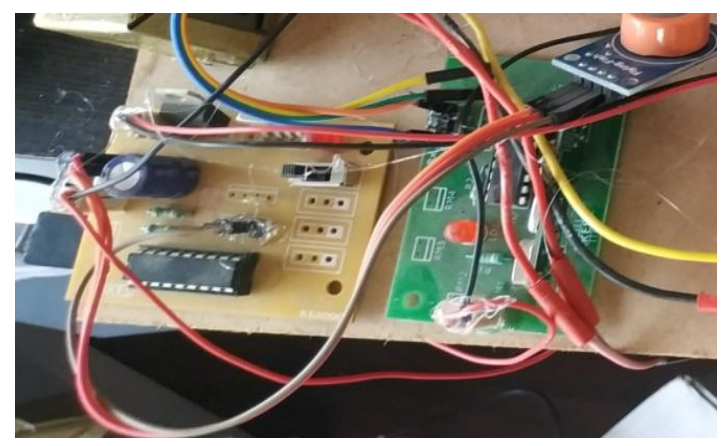

Helmet section

Published By: 


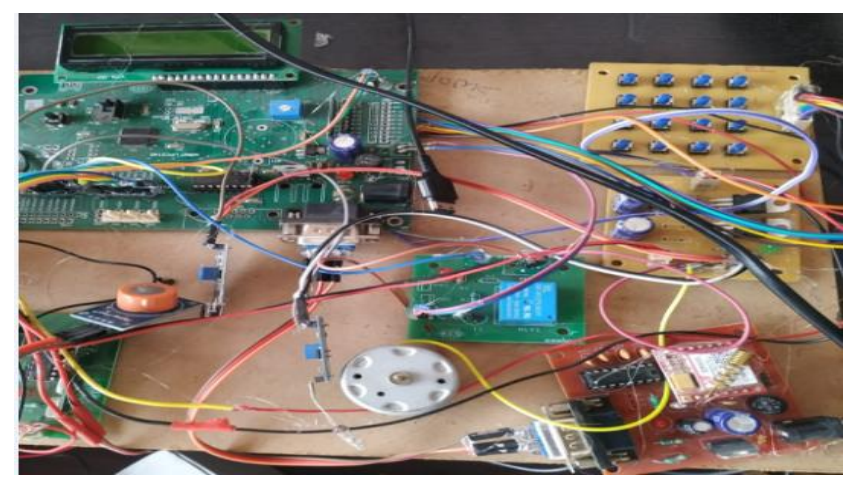

Vehicle section

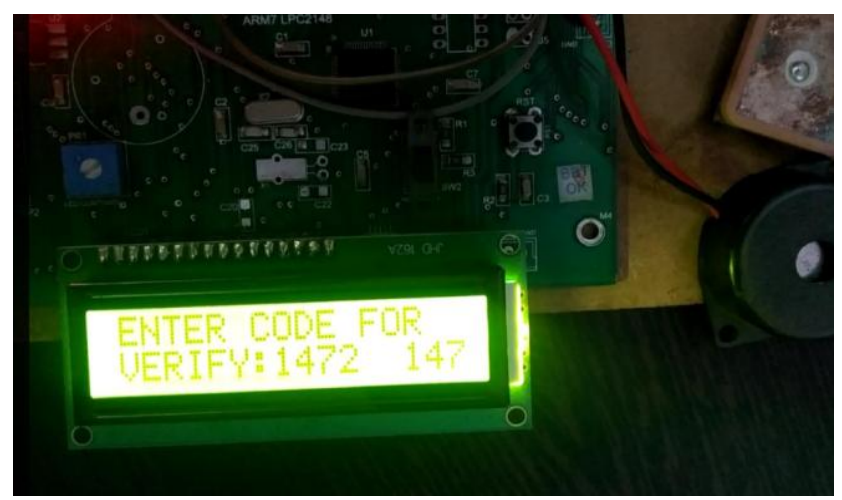

Verify the code suppose 1472

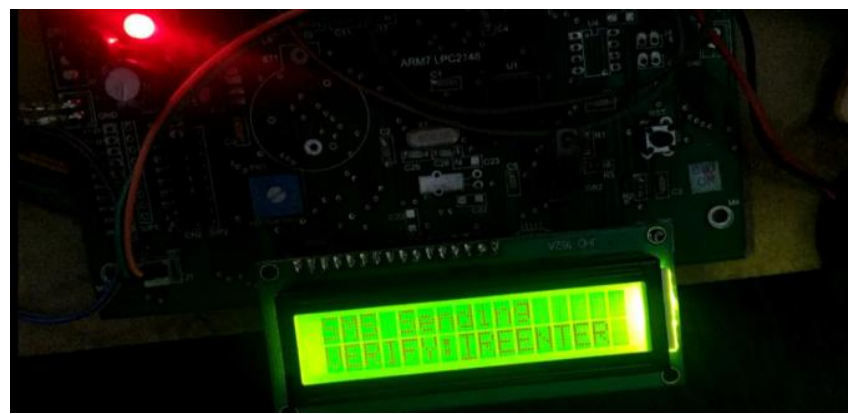

If the code is not correct SMS will sent to our mobile number as incorrect password

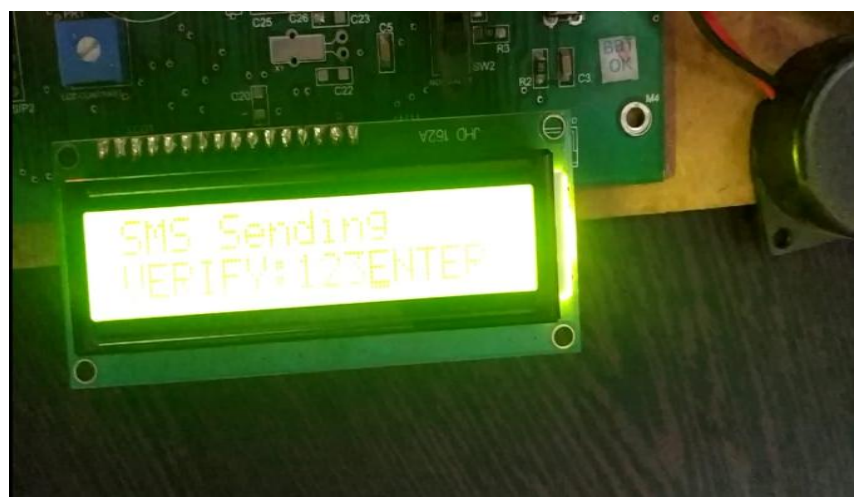

Again enter the password 1234. it will again send the sms to mobile number

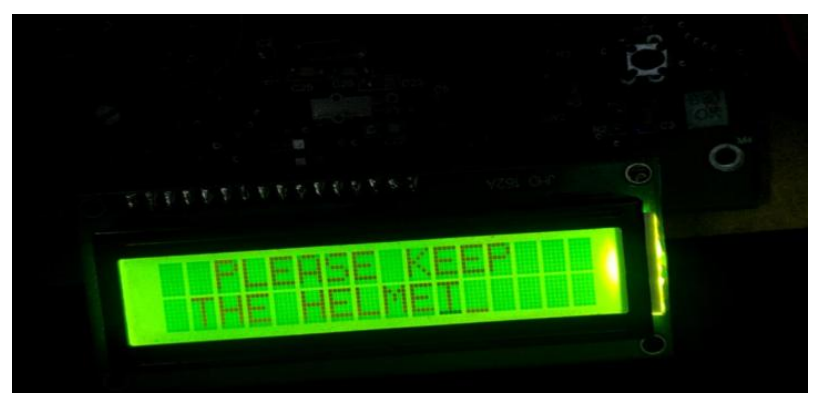

If the rider is not wear the helmet, then it display the sms as the keep helmet

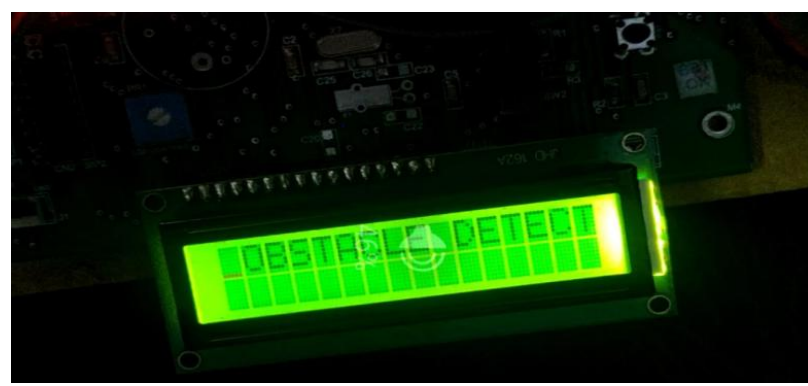

Obstacle detection also providing

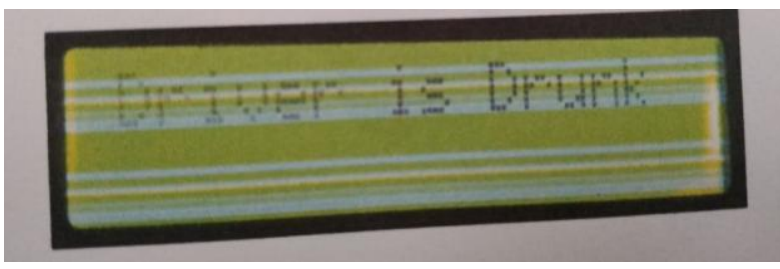

On the off chance that liquor fixation present in human breath, at that point it show the message on LCD and it sends the SMS to enlist versatile number with their present area.

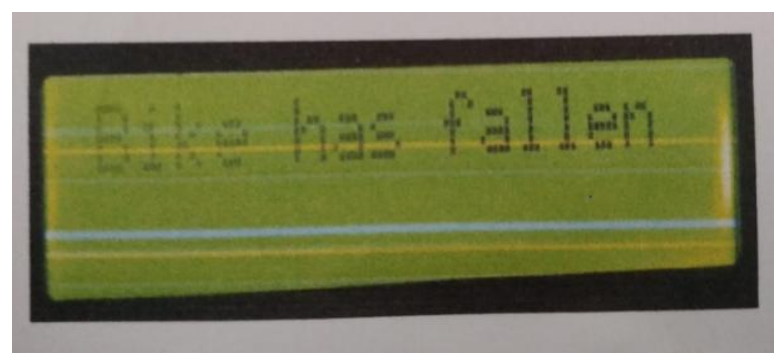

On the off chance that accidents happened, at that point bike is fallen. It shows the message on LCD. What's more, it sends the SMS to enroll number with their current location.

\section{CONCLUSIONS}

From this project it was concluded that the bike starts when we wear the helmet and this type of arrangement regarding the wearing of the helmet help us in minimizing the risks during the accidents.

This system requires the following technology such as GSM, GPS, RF communication. \& Sciences Publication
Blue Eyes Intelligence Engineering

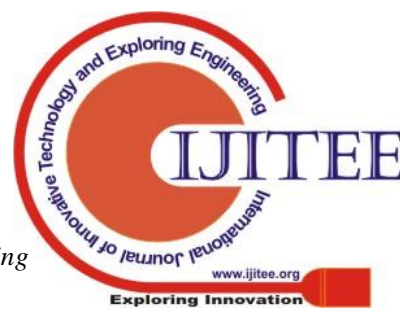


In this extension we can add some other additional sensor technology to maintain the safety of rider. And another important aspect of this project is providing the continuous GPS tracking system with the keypad technology.

\section{REFERENCES}

1. Prajitha Prasad A1, Reshma Mohan2, "smart helmet and intelligent bike system", ISSN (PRINT): 2393-8374, (ONLINE): 2394-0697, VOLUME-5, ISSUE-5, 2018.

2. Bhumi Bhatt1, Purvi Kalani2 "Smart Vehicle Security System Using GSM \& GPS", International Journal Of Engineering And Computer Science ISSN:2319-7242 Volume 4 Issue 6 June 2015, Page No. 12508-12511.

3. S. S.Pethakar, N.Srivastava, and S.D.Suryawanshi,"RFID, GPS and GSM based vehicle tracing andemployee security system,"International Journal ofAdvanced Research in Computer Science andElectronics Engineering, vol. 1, no. 10, pp. 91-96,Dec.2012.

4. P. P. Wankhade and S.O. Dahad, "Real timevehicle locking and tracking system using GSM andGPS technology-an antitheft system," InternationalJournal of Technology and Engineering System, vol.2, no. 3, p.272-275, March 2011.

5. J.R. Shaikh and S. M. Kate, "Arm7 based smartcar security system," International Journal ofEngineering Trends and Technology, vol. 3, no. 2,pp. 210-212, March 2012 